MS37-04

\section{Crystal adaptronics: mechanically reconfigurable elastic and superelastic molecular crystals}

Pance Naumov $^{1}$, Ejaz Ahmed ${ }^{1}$, Durga Prasad Karothu ${ }^{1}$

1. New York University Adbu Dhabi, Abu Dhabi, United Arab Emirates

email: pance.naumov@nyu.edu

Mechanically reconfigurable molecular crystals - ordered materials that can adapt to variable operating and environmental conditions by deformation, whereby they attain motility or perform work - are quickly shaping up a new research direction in materials science, crystal adaptronics. Properties such as elasticity, superelasticity and ferroelasticity that are normally related to inorganic materials, and phenomena such as shape-memory and self-healing effects which are well established for soft materials, are increasingly reported for molecular crystals, yet their mechanism, quantification, and relation to the crystal structure in organic crystals are not immediately intelligible to the chemistry and materials science research communities. This talk will provide a condensed topical overview of the elastic, superelastic and ferroelastic molecular crystals, emerging new classes of materials that bridge the gap between the soft matter and inorganic materials. The occurrence and detection of these unconventional properties, and the underlying structural features of the related molecular materials will be discussed and highlighted together with selected prominent recent examples.

References:

[1] Ahmed, E., Karothu, D. P. \& Naumov, P. (2018) Angew. Chem. Int. Ed., doi: 10.1002/anie.201800137Keywords: mechanical effects, elasticity, actuators

Keywords: mechanical effects, elasticity, actuators
MS37-05

\section{Mechanochemistry, a tool for improving drug physicochemical properties and delivery: salt formation and metal coordination}

Maria Teresa Duarte ${ }^{1}$, Inês C.B. Marins ${ }^{1}$, Sílvia Quaresma ${ }^{1}$, Vânia André ${ }^{1}$

1. Centro de Química Estrutural, Instituto Superior Técnico, Lisboa, Portugal

email: teresa.duarte@tecnico.ulisboa.pt

Mechanochemical strategies were used for solving folic acid solubility issues as well as improving gabapentin polymorphic stability. Biological active Metal Organic Frameworks were synthesized and proved to be promising for drug delivery as the release is achieved through the degradation of the solid. Structures were determined by X-ray powder and single crystal diffraction and SSNMR. MD calculations were used to ascertain experimental results obtained both when controlling the stability of polymorphs as well as attesting SS-NMR results.

This results were obtained in our group when developing strategies to enhance poor aqueous solubility, low dissolution rate and low bioavailability, well known problems of $40 \%$ of the approved Active Pharmaceutical Ingredients (APIs) in the market.

Similarly, we have been addressing the use of metal organic frameworks, based on safe metals and drug based linkers to attain controlled delivery. The API is part of the matrix, avoiding multistep procedures to prepare the loaded material, porosity is no longer required.

References:

[1] Horcajada, P.; Gref, R.; Baati, T.; Allan, P.K.; Maurin, G.; Couvreur, P.; Férey, G.; Morris, R.E.; Serre, C. 2012, Chemical Reviews, 112, 1232-1268

[2] Martins, I.; Oliveira, M. C; Diogo, H.; Branco, L., Duarte, M. Teresa, 2017, ChemSusChem, 10 (7), 1360-1363

[3] Martins, I.; Gomes, J., Duarte, M. Teresa; Mafra, L., 2017, Crystal Growth and Design, 17 (2), 428-432

Keywords: mechanochemistry, salt formation, metal organic frameworks 